\title{
Functionalised Nanostructured Polyaniline - A New Substrate for Building Adaptive Sensing Surfaces
}

Emer Lahiff ${ }^{1}$, Steven Bell ${ }^{2}$, and Dermot Diamond ${ }^{1}$

${ }^{1}$ National Centre for Sensor Research, Dublin City University, Dublin 9, Ireland

${ }^{2}$ Queen's University Belfast, University Road, Belfast, BT7 1NN, Ireland

\begin{abstract}
A new method for covalently binding side-chains to the surface of solution based conducting polymer nanostructures is introduced in this paper. Modification of the structures is achieved by convenient reflux in the presence of a nucleophile, and post-functionalisation purification is subsequently carried out by centrifugation. The entire process is easily scalable and hence suitable for bulk production of functionalised nanomaterials. In particular we focus on the modification of polyaniline nanofibres which can be synthesized by interfacial polymerization. Mercaptoundecanoic acid side-chains are attached to the polymer nanostructures, with the intrinsic nano-morphology of the material being maintained during the process. The modified PAni nanofibres provide a template for the attachment of other specific functional groups which could be used to target a particular species.
\end{abstract}

\section{INTRODUCTION}

Inherently conducting polymers (ICPs) can be used in applications which exploit their tunable conductivity. Many ICPs are however unstable and degrade when exposed to atmospheric conditions. Polyaniline (PAni) is an example of a stable ICP whose conductivity can be controlled over ten orders of magnitude by changing the environment of the material. PAni switches reversibly between an insulating emeraldine base form and a conducting emeraldine salt form. Consequently PAni has huge potential in sensor applications ${ }^{1-3}$. By adapting the morphology of PAni, we can increase the surface area exposed to target molecules. In this work we focus on PAni nanofibre structures which can be synthesised in a simple one-step process by interfacial polymerisation ${ }^{4-6}$. In particular, we will demonstrate how this material can be functionalised. Using the technique described, novel multifunctional materials, whose properties can be externally tuned and controlled, can be produced. These can be classed as adaptive materials in that they are systems which are capable of reacting in an intelligent way to changes in their environmental conditions. The resulting functionalised conducting polymer is characterised using electron microscopy, cyclic voltammetry and spectroscopic techniques (Fourier Transform infrared and Raman).

\section{EXPERIMENTAL DETAILS}

Polyaniline nanofibres were synthesised under ambient conditions by interfacial polymerisation between an aqueous and an organic layer. The aqueous layer contained $1 \mathrm{M}$ 
hydrochloric acid as the dopant acid and ammonium peroxydisulfate $\left(2-60 \mathrm{mmol},\left(\mathrm{NH}_{4}\right)_{2} \mathrm{~S}_{2} \mathrm{O}_{8}\right)$ as the oxidising agent. The organic layer contained purified aniline (40-200 mmol) dissolved in toluene. Green polyaniline appeared initially at the interface and then migrated into the aqueous phase, after 24 hours the reaction was complete. The product was purified by centrifugation (300 $\mathrm{rpm} / 10 \mathrm{~min} / 3$ cycles) and suspended as a colloid in deionised water. PAni nanofibres were subsequently modified with mercaptoundecanoic acid (MA) by refluxing at $100^{\circ} \mathrm{C}$ for 2 hours in an aqueous $\mathrm{pH} 4$ buffer. The product was purified by centrifugation $(300 \mathrm{rpm} / 10 \mathrm{~min} / 3$ cycles $)$ and could be re-suspended as a colloid in deionised water.

Nanofibre morphology was studied using field emission scanning electron microscopy (FESEM) at an accelerating voltage of $20 \mathrm{kV}$ on a S-4300 Hitachi system. Samples were cast as films onto silicon wafers and coated with $10 \mathrm{~nm} \mathrm{Au} / \mathrm{Pd}$ prior to imaging. The bonding of MA side-chains to PAni nanofibres was investigated using a variety of techniques. Cyclic voltammetry experiments were carried out using a $\mathrm{CH}$ Instruments electrochemical analyser. Scans were taken in $0.1 \mathrm{M} \mathrm{KCl}$ electrolyte at a rate of $50 \mathrm{mV} / \mathrm{sec}$ from $-0.4 \mathrm{~V}$ to $0.8 \mathrm{~V}$. A glassy carbon electrode ( $\mathrm{CH}$ Instruments, $3.5 \mathrm{~mm}$ diameter) was used as a substrate (working) electrode. The counter electrode was a platinum wire and the reference electrode was $\mathrm{Ag} / \mathrm{AgCl}$. Fourier Transform infrared spectra (FTIR) were recorded in transmission mode from 400 to $4000 \mathrm{~cm}^{-1}$, for 100 repeat scans, with a resolution of $8 \mathrm{~cm}^{-1}$ and at $1 \mathrm{~cm}^{-1}$ intervals on a Perkin Elmer Spectrum GX FTIR instrument. Samples were dried under vacuum at $50^{\circ} \mathrm{C}$ for 4 hours prior to FTIR spectra being acquired, and then mixed with dried KBr. Raman spectroscopy was carried out on an Avalon 785nm Raman spectrometer at $2 \mathrm{~cm}^{-1}$ resolution, 3 seconds per scan and 10-20 collections. A $785 \mathrm{~nm}$ laser line was used as it can detect both doped and de-doped PAni features.

\section{DISCUSSION}

Interfacial polymerization resulted in the formation of polyaniline nanofibres (Figure 1A). For the aniline:oxidant ratios used to synthesise the PAni precursor the nanofibre yield scales linearly with volume, and for a ratio of 200:60 mmol the yield varied at a rate of approximately $4 \mathrm{mg} / \mathrm{ml}$ (this was measured for total reaction volumes in the range 40 to $400 \mathrm{ml}$ ). The fibres were modified with MA and purified post-reflux by centrifugation. After the third rinse, the supernatant was clear indicating that all non-covalently bound material had been removed. Purified PAni-MA fibres could be re-dispersed in solvents such as deionised water to form colloid suspensions. Similar to PAni, PAni-MA could be cast as films onto substrates such as silicon. When imaged using FESEM it was observed that the nano-morphology was unaffected by functionalisation (Figure 1B). 

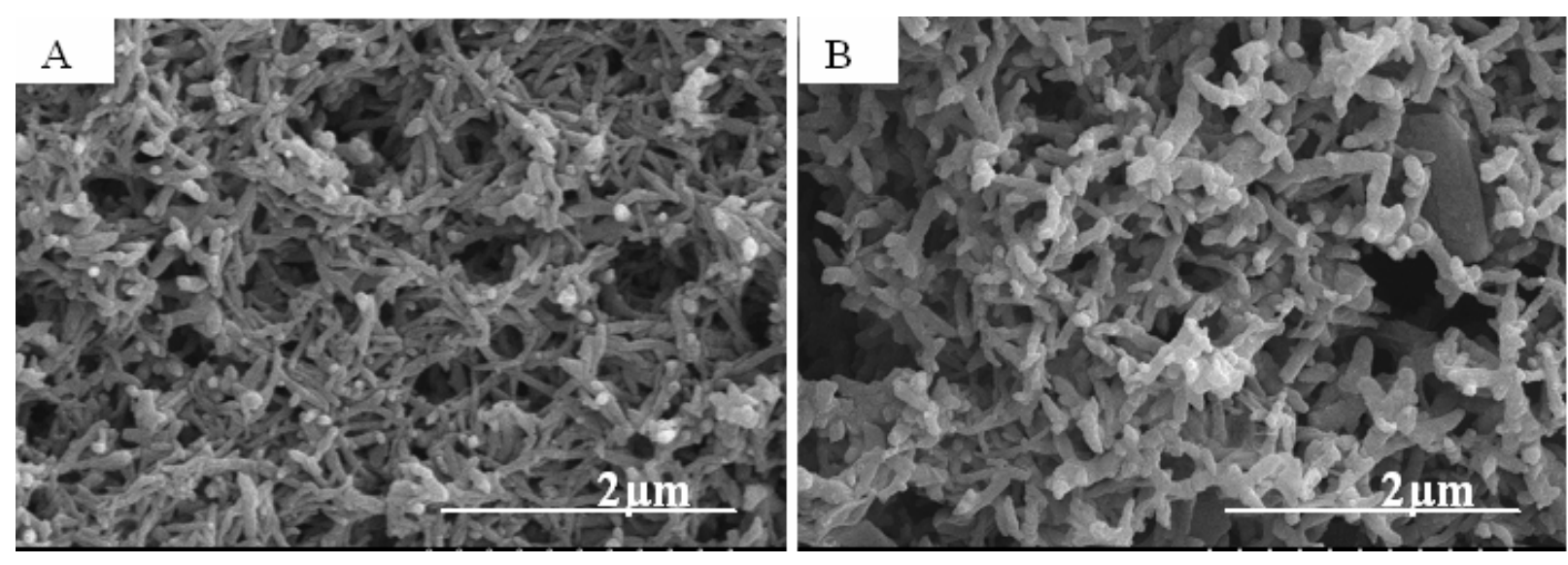

Figure 1. Polyaniline nanofibre morphology (A) is maintained post-functionalisation (B). Fibre diameters ranged from 40-80 nm (including a $10 \mathrm{~nm}$ conductive coating deposited for imaging purposes).

Higher resolution FESEM images show an increase in surface roughness of nanofibres postreflux suggesting that functionalisation may have been successful. This was investigated further using FTIR. A spectrum of MA $\left(\mathrm{HS}\left(\mathrm{CH}_{2}\right)_{10} \mathrm{COOH}\right)$ powder revealed the expected peaks and some of these are listed in Table 1. For FTIR spectra of PAni-MA, characteristic MA peaks appeared alongside typical PAni peaks indicating that functionalisation had been successful. Closer inspection showed that in fact certain signature peaks had shifted for the PAni-MA compared to the MA powder, suggesting covalent attachment (Table 1). The most significant peaks, when considering covalent attachment, are the thiol (S-H) bonds. These appear at $489 \mathrm{~cm}^{-}$ ${ }^{1}, 706 \mathrm{~cm}^{-1}$ and $2556 \mathrm{~cm}^{-1}$ and all disappear in the functionalised material, in agreement with covalent attachment of the reactive thiol nucleophile to an electrophilic quinoid ring on the PAni backbone ${ }^{7}$.

Table 1. FTIR peak shifts indicate covalent bonding.

\begin{tabular}{|l|l|l|}
\hline MA & $\begin{array}{l}\text { PAni-MA } \\
\left(\mathrm{cm}^{-1}\right)\end{array}$ & Peak Assignments \\
\hline 489 & gone & $\mathrm{C}-\mathrm{S}-\mathrm{H}$ out-of-plane bend \\
\hline 686 & 683 & $\mathrm{C}-\mathrm{S}$ \\
\hline 706 & gone & $\mathrm{C}-\mathrm{S}-\mathrm{H}$ in-plane bend \\
\hline $723 / 730$ & $720 / 733$ & $\mathrm{C}-\mathrm{S}$ (doublet) \\
\hline 1105 & 1107 & $\mathrm{C}-\mathrm{C}$ alkyl stretch \\
\hline 1211 & 1208 & $\mathrm{CH}_{2}$ \\
\hline 1238 & 1233 & $\left.\mathrm{C}-\mathrm{C}^{-1}\right)$ \\
\hline 1264 & 1264 & $\mathrm{CH}_{2} \mathrm{~S}$ \\
\hline 1701 & $1696 / 1712$ & $\mathrm{C}=\mathrm{O}$ (splits) \\
\hline 2556 & gone & $\mathrm{S}-\mathrm{H}$ \\
\hline
\end{tabular}




\section{Cyclic Voltammetry}

Cyclic voltammetry of PAni nanofibres showed a redox profile characteristic of PAni ${ }^{8}$ (Figure 2). The first oxidation peak was observed at $0.19 \mathrm{~V}\left(\mathrm{I}=4 \times 10^{-4} \mathrm{~A}\right)$ with a second oxidation peak at $0.580 \mathrm{~V}\left(\mathrm{I}=4 \times 10^{-4} \mathrm{~A}\right)$. On reversing the bias, PAni is reduced, as $\mathrm{H}^{+}$ions are expelled. Reduction peaks occur at $0.32 \mathrm{~V}\left(\mathrm{I}=3 \times 10^{-4} \mathrm{~A}\right)$ and $0.10 \mathrm{~V}\left(\mathrm{I}=3 \times 10^{-4} \mathrm{~A}\right)$. Scans on functionalized PAni-MA nanofibres were carried out under the same conditions. The CV profile differs from that of unmodified PAni as only one redox peak occurs (Figure 2). The oxidation peak is at $0.42 \mathrm{~V}\left(\mathrm{I}=5 \times 10^{-6} \mathrm{~A}\right)$ with a single reduction peak at $0.12 \mathrm{~V}\left(\mathrm{I}=4 \times 10^{-6} \mathrm{~A}\right)$. The redox cycling for PAni-MA is reversible and the current flow for both oxidation and reduction is equal, but less than the current flow for PAni. The electrochemical profile therefore differs noticeably from that of PAni, suggesting that the available redox states have been altered.
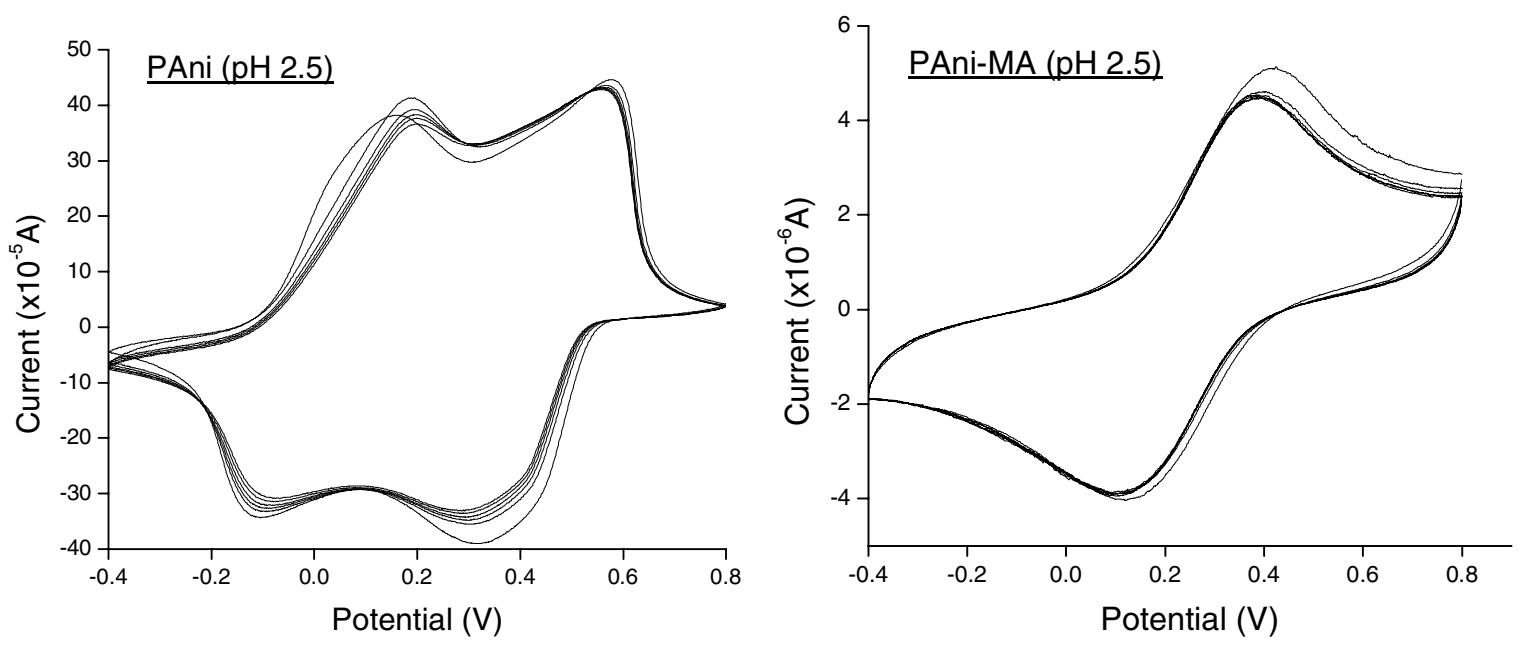

Figure 2. Cyclic voltammetry shows that the functionalised PAni-MA is electrochemically active and that redox cycling is reversible. The profile differs, however, from that for unmodified PAni suggesting that the material has been altered.

\section{$\underline{\text { Raman Spectroscopy }}$}

Raman spectra of both PAni and PAni-MA show similar spectral changes as a response to changes in the $\mathrm{pH}$ of the environment. For low $\mathrm{pH}$ values the polymer exists in the doped, conductive state. Increasing the $\mathrm{pH}$ causes in a change in the bonding structure of the material and results in a de-doped, insulating material. This change in structure can be detected by Raman spectroscopy. Signature bands between $1300-1400 \mathrm{~cm}^{-1}$ appear for the doped material (Figure $3 \mathrm{~A})$. These are less significant for the de-doped material. Strong bands between $1400-1500 \mathrm{~cm}^{-1}$ are characteristic of the de-doped material (Figure 3B). In particular, peaks at $1340 \mathrm{~cm}^{-1}$ can be assigned to a $\mathrm{C}-\mathrm{N}^{\bullet+}$ polaronic stretch, which is characteristic of the doped state ${ }^{9,10}$. Peaks at $1490 \mathrm{~cm}^{-1}$ are characteristic of $\mathrm{C}=\mathrm{N}$ quinoid groups and indicate de-doping ${ }^{10}$. Raman evidence therefore indicates that the intrinsic redox properties of PAni are maintained postfunctionalisation. 

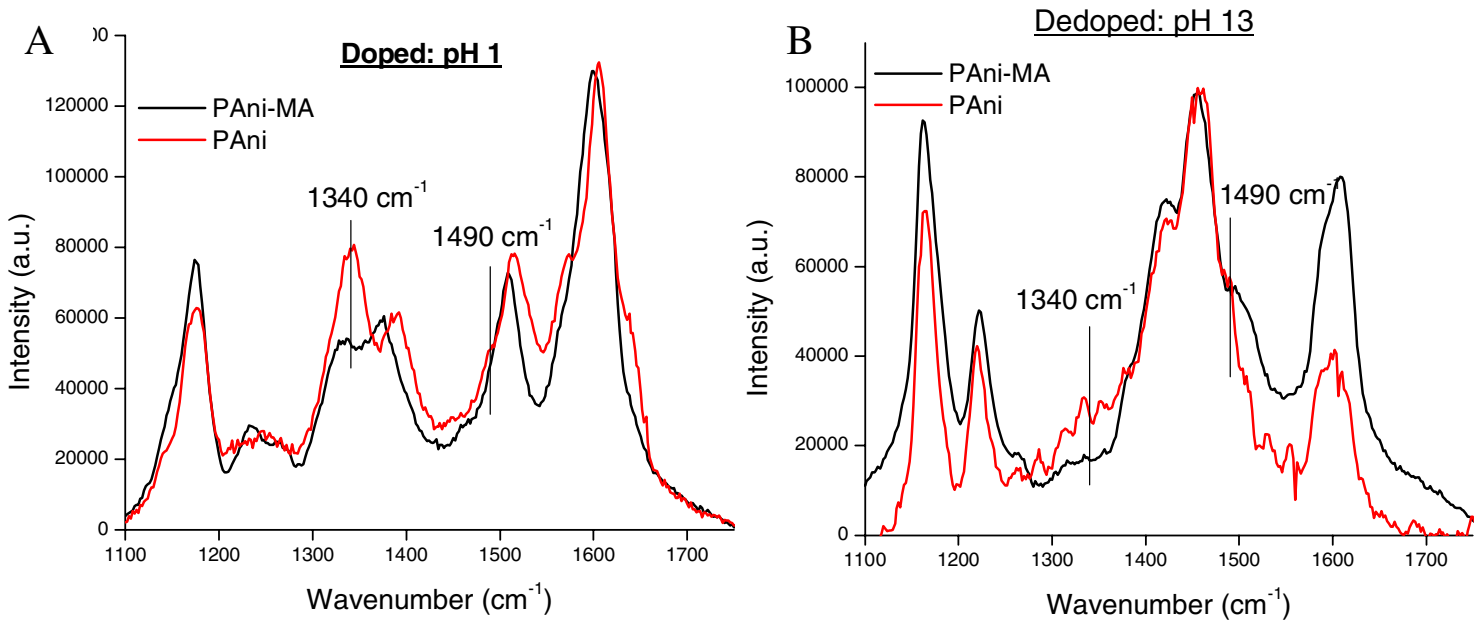

Figure 3. Raman spectra show that functionalised PAni-MA nanofibres can be doped and dedoped controllably (similar to PAni) by altering the $\mathrm{pH}$ of the environment.

\section{CONCLUSIONS}

This study shows the successful functionalisation of solution based PAni nanostructures. Modified fibres can be easily purified by centrifugation and re-suspended in solvents to form colloids. By focusing on the modification of nanofibre surfaces post-polymerisation, the intrinsic properties (such as doping) of the PAni backbone can be maintained. The modified PAni nanofibres provide a scaffold which could be further modified with selective moieties for the detection of target species. Using the process described, we can combine the advantages of a functionalised organic conductor and a low dimensional nanostructure. The technique described is simple, cost-effective and easily scalable.

\section{ACKNOWLEDGEMENTS}

The authors acknowledge funding from Science Foundation Ireland under the 'Adaptive Information Cluster' award no. SFI 03/IN.3/1361. The authors also thank Dr. Benjamin Schazmann (Dublin City University) and Prof. Leon Kane-Maguire (University of Wollongong, Australia) for helpful discussions. 


\section{REFERENCES}

1 S. Virji, J. X. Huang, R. B. Kaner, and B. H. Weiller, Nano Letters 4, 491-496 (2004).

S. Virji, R. B. Kaner, and B. H. Weiller, Chemistry of Materials 17, 1256-1260 (2005).

3 S. Virji, R. B. Kaner, and B. H. Weiller, Journal of Physical Chemistry B 110, 2226622270 (2006).

4 J. X. Huang and R. B. Kaner, Journal of the American Chemical Society 126, 851-855 (2004).

5 J. X. Huang, S. Virji, B. H. Weiller, and R. B. Kaner, Journal of the American Chemical Society 125, 314-315 (2003).

N. R. Chiou and A. J. Epstein, Synthetic Metals 153, 69-72 (2005).

7

C. Barbero, G. M. Morales, D. Grumelli, G. Planes, H. Salavagione, C. R. Marengo, and M. C. Miras, Synthetic Metals 101, 694-695 (1999).

A. Talaie, J. Y. Lee, Y. K. Lee, J. Jang, J. A. Romagnoli, T. Taguchi, and E. Maeder, Thin Solid Films 363, 163-166 (2000).

R. Mazeikiene, A. Statino, Z. Kuodis, G. Niaura, and A. Malinauskas, Electrochemistry Communications 8, 1082-1086 (2006).

C. Liu, J. X. Zhang, G. Q. Shi, and F. E. Chen, Journal of Applied Polymer Science 92, 171-177 (2004). 